\title{
Erratum to: Carga de la enfermedad y costes asociados del asma no grave en adultos en España: análisis de datos de una comunidad representativa
}

\author{
Antoni Sicras-Mainar ${ }^{1}$ - Jesús I. Diago-Cabezudo ${ }^{2}$ - Guadalupe Sánchez-Herrero ${ }^{2}$. \\ Ruth Navarro-Artieda ${ }^{3}$ - Manuel García-Losa ${ }^{4}$
}

Published online: 20 May 2016

(C) Springer International Publishing Switzerland 2016

Erratum to: Pharmacoecon Span Res Artic

DOI 10.1007/s40277-016-0058-6

Existe una errata en el apellido del último autor citado en este artículo. El nombre completo correcto es: Manuel GarcíaLosa.
En el apartado de conflicto de intereses, se añade uno de los autores:

“Jesús I. Diago-Cabezudo y Guadalupe Sánchez-Herrero son empleados de GSK. Los demás autores declaran no tener conflicto de intereses".

The online version of the original article can be found under doi:10.1007/s40277-016-0058-6.

$凶$ A. Sicras-Mainar asicras@bsa.cat

1 Dirección de Planificación, Badalona Serveis Assistencials, Via Augusta, 9, 08911 Badalona, Barcelona, España

2 Departamento Médico, GlaxoSmithKline, Tres Cantos, Madrid, España

3 Documentación Médica, Hospital Germans Trias i Pujol, Badalona, Barcelona, España

4 Health Economics and Outcomes Research, IMS Health, Barcelona, España 